Revista PSICOLOGIA, 2020, Vol. 34 (1), 67-81. doi: 10.17575/psicologia.v34i1.1473

\title{
Trabalho em call-center: Comparação entre os serviços inbound e outbound
}

\author{
Susana Gonçalves ${ }^{1}$, Isabel Soares Silva ${ }^{1}$ \& Ana Veloso ${ }^{1}$ \\ ${ }^{1}$ Escola de Psicologia da Universidade do Minho, Campus de Gualtar, 4710-057 Braga, Portugal.
}

\begin{abstract}
Resumo: 0 impacto do trabalho em call-center em várias dimensões físicas e psicológicas do indivíduo tem sido alvo de diversos estudos; no entanto, pouca investigação tem comparado os tipos de serviços neste contexto de trabalho. Assim, este estudo, além de fazer uma análise das características do trabalho, das principais fontes de stress e do compromisso com a organização neste tipo de contexto, comparou os serviços inbound e outbound em função de tais dimensões. No total, participaram 274 operadores de callcenter, 191 do serviço inbound e 83 do serviço outbound. Na recolha de dados foi usado um protocolo de investigação constituído por diversas escalas, em formato papel e online. Verificou-se que participantes do grupo inbound percecionam uma maior variedade de competências no seu trabalho e níveis superiores de stress relacionados com problemas familiares do que os participantes do grupo outbound. Não foram encontradas diferenças significativas em relação ao compromisso com a organização.
\end{abstract}

Palavras-chave: Call-center; características do trabalho; stress; compromisso organizacional.

Work in call-center: Comparison between inbound and outbound services. The impact of call-center work on various physical and psychological dimensions of the individual has been subject of several studies; however, research comparing the types of services in this work context is scarce. Thus, in this study, besides analysing the characteristics of the work, the main sources of stress and the organizational commitment in this type of context, also compared the inbound and outbound services according to such dimensions. In total, 274 call-center operators participated, 191 of the inbound service and 83 of the outbound service. In the data collection the protocol used consisted in several scales, in paper format and online. It was found that participants in the inbound group perceive a greater variety of skills in their work and higher levels of stress related to family problems than the participants of the outbound group. No significant differences were found relatively to the commitment to the organization.

\section{Keywords: Call-center; work characteristics; stress; organizational commitment.}

As constantes alterações das sociedades, bem como o avanço tecnológico, potenciaram o estudo de novas estratégias de trabalho. A promoção de serviços e o apoio a clientes através de meios telefónicos e informáticos foi uma das estratégias de trabalho adotadas nas últimas décadas, fazendo assim emergir os denominados call-centers.

Os call-centers caracterizam-se pela promoção, entrega e venda de produtos/serviços por via telefónica e por meio da utilização de um terminal de computador, de forma ágil, rápida e com custos reduzidos para a organização (Legros et al., 2018; Mustosmäki et al., 2013; Sá \& Sá, 2014; Vilela \& Assunção, 2004; Zapf et al., 2003). 0 trabalho aí desempenhado é descrito como sendo feito sentado, de frente para um computador, com headset, e regidos por um protocolo empresarial desenhado de forma a maximizar o número de chamadas efetuadas (e.g., Holman et al., 2002; Moreira et al., 2015; Rose \& Wright, 2005). Esta forma contemporânea de trabalho promove a facilidade no contacto entre empresas e os seus clientes, o que fez aumentar significativamente o número de call-centers (Echchakoui, 2016; Roque, 2013; Scolari et al., 2009). 0 crescimento significativo do número de call-centers tem chamado a atenção da comunidade científica e este interesse não surge apenas pela relativa novidade do setor de atividade, mas também pelas questões do âmbito da segurança e saúde no trabalho que tal temática suscita (Moreira et al., 2015).

De acordo com o European Contact Center Benchmark realizado em 30 países europeus no ano 2015, o número de call-centers tem vindo a apresentar uma taxa de crescimento de $3.02 \%$ ao ano, o que significa um aumento do número de operadores neste tipo de serviços. Nesse ano, existiam na Europa perto de 40000 call-centers que empregavam mais de 3,85 milhões de pessoas (1.15\% da população ativa

${ }_{1}^{1}$ Morada para correspondência: Isabel Soares Silva, Escola de Psicologia da Universidade do Minho, Campus de Gualtar, $4710-057$ Braga, Portugal. E-mail: isilva@psi.uminho.pt 
da Europa), sendo que a maioria desses profissionais são do sexo feminino (64.4\%), 42.7\% possuem o ensino secundário e 66.4\% trabalham a tempo inteiro no call-center (Altitude, 2016). Em Portugal, comparados os dados recolhidos em 2013 no European Contact Center Benchmark com os de 2014, a taxa de crescimento dos call-centers foi de $9 \%$.

Apesar de ser um tipo de atividade económica relativamente recente, a forma como o trabalho em call-center está organizado é considerado, por diversos autores, uma nova vertente do trabalho taylorizado (Costa \& Costa, 2018; Moreira et al., 2015; Sá \& Sá, 2014; Mustosmäki et al., 2013; Santos \& Marques, 2006; Vilela \& Assunção, 2004). A organização do trabalho taylorizado, proposta por Taylor (1911/1998), preconiza que o trabalho deveria estar organizado em tarefas simples, separado em pequenas atividades e distribuído por postos de trabalho de forma a especializar o operador naquela função e minimizar o tempo de realização da mesma, o que se assemelha ao que é pretendido nos serviços de call-center (Costa \& Costa, 2018; Moreira et al., 2015). De acordo com Santos e Marques (2006), os operadores de call-center são submetidos a uma monitorização constante feita de forma automática por parte de um supervisor direto que, através de software específico, acompanha e controla todo o desempenho em tempo real. A instalação de tal software permite conduzir as falas dos operadores, não admitindo alterações ou interpretações, tornando-as objetivas e padronizadas. Deste modo, todo o discurso do operador é limitado e controlado, reduzindo ao máximo a sua autonomia e o tempo de atendimento (Venco, 2006). Os operadores não têm autorização para determinar o momento e duração das chamadas, ficando sujeitos a tarefas rotineiras, de baixa complexidade, com reduzidas oportunidades para organizar o seu trabalho e a receção de feedback que, por vezes, é feito no decorrer da chamada (Costa \& Costa, 2018; Santos \& Marques, 2006; Roque, 2008). Os operadores de call-center consideram ainda estar sujeitos a um elevado ritmo de trabalho, justificado pelo reduzido intervalo de tempo entre chamadas, e a tarefas monótonas (Rose \& Wright, 2005).

Relativamente à forma como o trabalho está organizado nos serviços de call-center, Holman (2003) propõe dois modelos distintos. Por um lado, o trabalho pode ser orientado pelo modelo de serviço de elevado compromisso (high commitment service), caracterizando-o por ter operários qualificados, atendimento ao cliente personalizado e pela realização de tarefas complexas não controladas por entidades superiores. Por outro lado, pode seguir o modelo de serviço em massa (mass service), que o caracteriza por não necessitar de operários qualificados, por apresentarem baixa autonomia para os seus operadores na realização de tarefas pouco complexas, seguidas de processos previamente estabelecidos, propiciando assim um maior volume de chamadas. Conforme referido por Frenkel et al. (1998), a maioria dos call-centers consideram mais importante a quantidade de chamadas efetuadas/atendidas do que propriamente a qualidade do serviço. Também Costa e Costa (2018), num estudo comparativo do trabalho em call-center entre Portugal e Brasil, verificaram que em ambos os países, os rendimentos podem variar em função do número de vendas, o que vem sugerir que a maioria dos serviços de callcenter opta então por trabalhar de acordo com o segundo modelo proposto por Holman (2003), ou seja, o mass service.

Atendendo a que a literatura tem evidenciado uma ligação entre as características do trabalho em call-center e o bem-estar dos operadores (e.g., Holman, 2002; Scolari et al., 2009) alguns estudos têm focado a relação deste tipo de trabalho com variáveis relacionadas com o estado de saúde física e psicológica dos operadores. Assim, estudos realizados sobre este tema têm indicado que este tipo de serviços apresenta uma elevada pressão nos operadores e provocam dificuldades não só ao nível físico como ao nível psicológico, conduzindo a problemas de vinculação e identificação com a tarefa (Ziliotto \& Oliveira, 2014). Roque (2008), por exemplo, observou que a nível psicológico, os operadores apresentavam desmotivação para a vida social e familiar como principal consequência dos permanentes e elevados níveis de exigência e de stress. Moreira et al. (2015) analisaram também o impacto deste trabalho ao nível do stress nos operadores, verificando que estes, devido a estarem insatisfeitos com o conteúdo das suas funções e com as características organizacionais, rescindiam do seu contrato.

Outro aspeto a salientar em relação a este tipo de serviços é a forma como os operadores telefónicos interagem com os clientes. Esta interação pode ser feita através de três diferentes modelos (Chuchual et al., 2010). Um operador que apenas recebe chamadas de clientes com dúvidas ou queixas relacionadas com um dado produto/serviço é orientado pelo modelo inbound, enquanto um operador que tem de contactar diferentes pessoas para promover os produtos/serviços da organização (telemarketing), para fazer questionários ou, ainda, para solicitar donativos, é orientado pelo modelo outbound. No entanto, esta divisão pode não ser totalmente distinta, podendo os operadores ter de efetuar os dois modelos de chamadas (inbound e outbound), modelo que é designado como misto (Chuchual et al., 2010). Numa tentativa de diferenciar os serviços inbound (cultura de serviço) e outbound (cultura de vendas), Echchakoui (2016, p. 72) sistematizou a literatura e apresentou as principais características dos serviços inbound (cultura de serviço) e outbound (cultura de vendas), descritas na Tabela 1. 
Tabela 1. Caracterização dos serviços Inbound e Outbound

\begin{tabular}{|c|c|c|}
\hline & $\begin{array}{c}\text { Inbound } \\
\text { Cultura de serviço }\end{array}$ & $\begin{array}{l}\text { Outbound } \\
\text { Cultura de vendas }\end{array}$ \\
\hline Objetivo principal & $\begin{array}{l}\text { Serviço ao Cliente } \\
\text { Questionários de Assistência } \\
\text { Organização da assistência }\end{array}$ & $\begin{array}{l}\text { Vendas } \\
\text { Informar sobre futuras promoções ou } \\
\text { de produtos futuros }\end{array}$ \\
\hline Tarefas principais & $\begin{array}{l}\text { Responder a todas as chamadas de } \\
\text { inbound }\end{array}$ & $\begin{array}{l}\text { Vendas proactivas em todas as } \\
\text { chamadas de outbound } \\
\text { Criar experiência emocional de forma } \\
\text { proactiva com cada consumidor }\end{array}$ \\
\hline $\begin{array}{l}\text { Interação consumidor- } \\
\text { vendedor }\end{array}$ & Confrontação & Relação \\
\hline Competência & $\begin{array}{l}\text { Competência técnica } \\
\text { Escuta ativa } \\
\text { Conhecimento do produto e serviço } \\
\text { Competências informáticas }\end{array}$ & $\begin{array}{l}\text { Competência e aptidão para vendas } \\
\text { Habilidade de comunicação e atitude } \\
\text { proactiva } \\
\text { Conhecimento do produto e serviço } \\
\text { Competências informáticas }\end{array}$ \\
\hline Desempenho & $\begin{array}{l}\text { Prestação do serviço ao cliente } \\
\text { Satisfação do cliente } \\
\text { Custo/contacto por chamada } \\
\text { Taxa de abandono }\end{array}$ & $\begin{array}{l}\text { Vendas por produto ou serviço ou por } \\
\text { tipo de chamada } \\
\text { Vendas por chamada ou por hora } \\
\text { Vendas cruzadas } \\
\text { Satisfação do cliente } \\
\text { Fidelização do cliente }\end{array}$ \\
\hline Controlo & Maioritariamente comportamental & Maioritariamente resultados \\
\hline Supervisão das tarefas & Monitorização e avaliação & Coaching, monitorização e avaliação \\
\hline Motivação & Interação com o cliente & Base de comissão \\
\hline
\end{tabular}

Nota. Fonte: Retirado de "Addressing differences between inbound and outbound agents for effective call center management" de S. Echchakoui, 2016, Global Business and Organizational Excellence, 36, p. 72.

Os estudos citados até ao momento expõem certas características do trabalho em call-center, bem como o seu impacto nos operadores em geral. Porém, tendo em consideração os diferentes tipos de serviço - inbound ou outbound -, alguns estudos têm procurado apurar se o tipo de serviço tem influência no impacto que o trabalho em call-center tem nos operadores. Lin et al. (2010), compararam os dois tipos de serviço mencionados ao nível do stress experienciado no desempenho da profissão no sentido de identificar as principais fontes de stress. Os autores constataram que não existiam diferenças estatisticamente significativas entre os serviços inbound e outbound nas fontes de stress. No entanto, em termos descritivos, identificaram como principal fonte de stress para ambos os tipos de serviço "ter que lidar com clientes difíceis". Mais recentemente, também Khalid et al. (2013), recorrendo a uma escala de avaliação dos níveis de stress no trabalho, compararam os dois tipos de serviço e verificaram que os operadores do serviço inbound reportavam níveis mais elevados de stress geral. Os autores apontam como explicações para esta diferença o facto de estes operadores estarem mais expostos à hostilidade por parte do cliente, sendo que muitas das chamadas recebidas estão relacionadas com queixas destes. Igualmente, Rod e Ashill (2013) estudaram a magnitude da relação existente entre fontes de stress, exaustão emocional e realização pessoal nos serviços inbound e outbound. Em primeiro lugar, verificaram que a relação entre exaustão emocional e a despersonalização era significativamente superior para o serviço outbound, o que foi explicado pelo facto de tais operadores sujeitos a esse serviço estarem focados na venda. Adicionalmente, constataram que a relação entre a despersonalização e a baixa realização pessoal era significativamente mais elevada para o grupo inbound, resultado que foi justificado pelo facto desses operadores sentirem que tratam os clientes de uma forma impessoal.

Ainda a propósito da variável stress e como vimos na descrição anterior, enquanto alguns autores (e.g., Khalid et al., 2013) optam por avaliar os níveis de stress geral, outros (e.g., Lin et al., 2010), procuram identificar as potenciais fontes indutoras de tal resposta, constituindo o segundo caso o foco do nosso trabalho. Neste contexto, sublinhe-se também a diversidade de modelos explicativos do stress ocupacional; com efeito e, como referem Lorente \& Yeves (2016), “(...) desde as primeiras definições de stress laboral como excesso de exigências face à capacidade para enfrentá-las, desenvolveram-se diversos 
modelos concetuais de stress, baseados no princípio do equilíbrio entre as características do trabalho e as do trabalhador" (p. 72).

Outra variável que tem sido estudada no contexto deste tipo de trabalho com o intuito de compreender a elevada taxa de turnover existente nos serviços de call-center e a precariedade no trabalho, é o compromisso do trabalhador com a organização (Costa \& Costa, 2018; Roque 2008). De acordo com Meyer e Allen (1991), o compromisso organizacional corresponde ao estado psicológico que caracteriza a relação do trabalhador com a organização e que tem implicações na sua decisão de continuar como membro dela. Segundo o Modelo das Três Componentes do Compromisso Organizacional proposto pelos autores citados, os trabalhadores encontram-se comprometidos com a organização através de relações emocionais (compromisso afetivo), relações transacionais apoiadas num investimento pessoal que visa um determinado retorno (compromisso calculativo) e de sentimentos de obrigação e de dever moral para com a organização (compromisso normativo). Dos estudos realizados, os dados têm evidenciado que, no geral, há um baixo nível de compromisso por parte dos operadores de call-centers (Echchakoui, 2016; Maia, 2011; Paiva et al., 2015). No estudo realizado por Maia (2011), verificou-se que os operadores de um call-center português não se sentiam comprometidos com a organização em qualquer uma das três componentes analisadas. Por seu lado, Paiva et al. (2015), observaram que os operadores sentiam uma obrigação para se esforçar pela empresa, que estavam comprometidos de forma afetiva com a organização, mas que apresentavam níveis baixos de compromisso no que se refere ao sentido de obrigação moral em permanecer na organização. Visando perceber as razões que levam o operador a estar comprometido, ou não, com a organização, alguns autores têm apontado a importância de variáveis do contexto do trabalho, como a forma como o trabalho está organizado (Jaaron \& Backhouse, 2011) e o suporte recebido pela organização (Wu \& Liu, 2014), ao passo que outros têm salientado a relevância de variáveis do próprio indivíduo, tais como o capital psicológico e o envolvimento com a organização (Simons \& Buitendach, 2013), o contrato psicológico e a intenção de sair da organização (Dhanpat et al., 2018; Alcover et al., 2012). Echchakou (2016) observou uma relação positiva e significativa entre a identificação do trabalhador com a organização e a intenção de saída. Constatou ainda que os operadores inbound apresentam menor identificação com a organização quando comparados com os operadores outbound, apresentando como possível explicação para este resultado a diferença do controlo e da missão do serviço de inbound e outbound, isto é, os operadores inbound querem atender bem todos os clientes, no entanto, também sabem que devem fazer o maior número de chamadas por dia para otimizar a produtividade.

Dado o continuo aumento do número de call-centers e uma vez que este tipo de atividade tem sido indicado como geradora de elevados níveis de stress e insatisfação para com o emprego, continua a ser pertinente a realização de estudos nesta área. Assim, o presente estudo, além de fazer uma análise das características do trabalho, das principais fontes de stress e do compromisso com a organização neste tipo de contexto, pretende contribuir para o aprofundamento da compreensão das diferenças entre os serviços inbound e outbound, tendo para o efeito comparado tais serviços em função das variáveis mencionadas.

\section{MÉTODO}

\section{Participantes}

No total, participaram no estudo 292 operadores telefónicos de um call-center de telecomunicações localizado no Norte do país e inserido num modelo de trabalho mass service. No entanto, seis operadores não indicaram o tipo de serviço em que trabalhavam e 12 referiram que trabalhavam em ambos os serviços (i.e., inbound e outbound) e dado o interesse em proceder à comparação destes, as suas respostas também não foram consideradas. Deste modo, a amostra ficou constituída por 274 operadores telefónicos com idades compreendidas entre os 18 e os 55 anos $(M=28.20, D P=6.04)$.

Relativamente ao tipo de serviço que os operadores executavam, verificou-se que a maioria se encontrava afeta ao serviço inbound $(n=191,69.7 \%)$ e os restantes $83(30.3 \%)$ estavam inseridos no serviço outbound.

Na Tabela 2 é apresentada a caracterização da amostra ao nível sociodemográfico e profissional, considerando quer o total da amostra quer os dois tipos de serviços de call-center. No global, a amostra é constituída na sua maioria por participantes do sexo feminino (60.8\%), solteiros (70.5\%) e com um nível de escolaridade correspondente ao ensino secundário (61.0\%). Relativamente à situação profissional, a maioria dos participantes trabalhava a tempo inteiro (84.9\%) e encontrava-se na empresa entre 1 a 6 anos (71.3\%). De um modo geral, não se registaram diferenças assinaláveis em função do tipo de serviços nas dimensões avaliadas. 
Tabela 2. Caracterização Sociodemográfica e Situação Profissional

\begin{tabular}{|c|c|c|c|c|c|c|}
\hline \multirow[b]{2}{*}{ Variável } & \multicolumn{2}{|c|}{ Outbound } & \multicolumn{2}{|c|}{ Inbound } & \multicolumn{2}{|c|}{ Total } \\
\hline & $n$ & $\%$ & $n$ & $\%$ & $n$ & $\%$ \\
\hline \multicolumn{7}{|l|}{ Sexo } \\
\hline Feminino & 54 & 65.1 & 112 & 58.9 & 166 & 60.8 \\
\hline Masculino & 29 & 34.9 & 78 & 41.1 & 107 & 39.2 \\
\hline \multicolumn{7}{|l|}{ Idade } \\
\hline Média (desvio-padrão) & \multicolumn{2}{|c|}{$28.32(5.65)$} & \multicolumn{2}{|c|}{$28.12(6.33)$} & \multicolumn{2}{|c|}{$28.2(6.04)$} \\
\hline \multicolumn{7}{|l|}{ Estado Civil } \\
\hline Solteiro (a) & 48 & 57.8 & 143 & 76.1 & 191 & 70.5 \\
\hline Casado (a)/União de facto & 30 & 36.1 & 40 & 21.3 & 70 & 25.8 \\
\hline Divorciado (a) & 5 & 6.0 & 4 & 2.1 & 9 & 3.3 \\
\hline Viúvo (a) & 0 & 0.0 & 1 & 0.5 & 1 & 0.4 \\
\hline \multicolumn{7}{|l|}{ Nível de escolaridade } \\
\hline $3^{\text {o }}$ Ciclo do ensino básico & 3 & 3.7 & 16 & 8.6 & 19 & 7.1 \\
\hline Ensino secundário & 49 & 59.8 & 115 & 60.2 & 164 & 61.0 \\
\hline Licenciatura & 24 & 29.3 & 48 & 25.1 & 72 & 26.8 \\
\hline Mestrado & 4 & 4.9 & 7 & 3.7 & 11 & 4.1 \\
\hline Outro & 2 & 2.4 & 1 & 0.5 & 3 & 1.2 \\
\hline \multicolumn{7}{|l|}{ Situação profissional } \\
\hline Trabalho a tempo parcial & 8 & 9.8 & 33 & 17.4 & 41 & 15.1 \\
\hline Trabalho a tempo inteiro & 74 & 90.2 & 157 & 82.6 & 231 & 84.9 \\
\hline \multicolumn{7}{|l|}{ Antiguidade na empresa } \\
\hline Até 6 meses & 4 & 4.8 & 7 & 3.7 & 11 & 4.0 \\
\hline De 6 meses até 1 ano & 13 & 15.7 & 13 & 6.8 & 26 & 9.5 \\
\hline De 1 ano até 3 anos & 33 & 39.8 & 60 & 31.6 & 93 & 34.1 \\
\hline De 3 anos até 6 anos & 24 & 28.9 & 78 & 41.1 & 102 & 37.2 \\
\hline De 6 anos até 10 anos & 9 & 10.8 & 26 & 13.7 & 35 & 12.8 \\
\hline De 10 anos até 15 anos & 0 & 0.0 & 5 & 2.6 & 5 & 1.8 \\
\hline Há mais de 15 anos & 0 & 0.0 & 1 & 0.5 & 1 & 0.4 \\
\hline
\end{tabular}

Nota. Dada a presença de valores omissos em certas variáveis, o $\mathrm{n}$ obtido pode diferir do tamanho da amostra $(\mathrm{N}=274)$.

\section{Instrumentos}

Os dados foram recolhidos através de um protocolo de investigação, integrando várias medidas que passamos a descrever.

Questionário sociodemográfico e profissional. 0 questionário sociodemográfico e profissional visou recolher informação sobre os participantes e sobre o seu local de trabalho, por forma a caraterizar a amostra (e.g., sexo, idade, nível de escolaridade, tipo serviço).

Características do trabalho. Para avaliar as características do trabalho foi usada a versão revista por Silva e Keating (2011) do Job Diagnostic Survey (Hackman \& Oldhan, 1976) a partir das recomendações da literatura (e.g., Boonzaier et al., 2001; Harvey et al., 1985; Idaszak \& Drasgow, 1987) com vista a melhorar as propriedades psicométricas do instrumento, nomeadamente a reformulação dos itens negativos em itens positivos e a operacionalização de âncoras em todos os pontos da escala. A escala inclui 15 itens divididos em duas secções e respondidos numa escala de Likert de sete pontos, sendo que quanto maior a pontuação obtida numa dada característica do trabalho, maior a perceção da sua presença por parte do trabalhador. Os itens estão organizados em cinco subescalas (três itens por subescala), nomeadamente: (i) "Variedade de Competências", que avalia o grau em que determinado trabalho requer a concretização de tarefas muito variadas, exigindo conhecimentos muito diferentes e complexos; (ii) "Identidade da tarefa", que refere que o trabalho do operador implica que seja feito do princípio ao fim por si, com um resultado visível; (iii) "Significado da tarefa", que descreve o impacto que determinado trabalho tem na 
vida ou trabalho de outras pessoas, quer no contexto organizacional quer no contexto externo; (iv) "Autonomia", que descreve o grau de liberdade que determinado trabalhador tem para realizar as suas tarefas de forma independente e com poder para planear e organizar o trabalho conforme a sua decisão e (v) "Feedback", que refere o grau de informação que determinado trabalhador recebe sobre a realização do seu trabalho.

Atendendo aos objetivos que nortearam o presente estudo bem como a preocupação de não ter um protocolo de investigação muito extenso, optou-se por incluir três das cinco subescalas, designadamente as seguintes: "Autonomia" (e.g., "Quanta autonomia existe no seu trabalho? Ou seja, até que ponto o seu trabalho é decidido por si?"), "Variedade de competências" (e.g., "Que variedade existe no seu posto de trabalho? Ou seja, até que ponto tem de fazer tarefas diferentes que exigem conhecimentos diferentes?") e "Feedback" (e.g., "Independentemente do que possam dizer os seus colegas ou chefias, até que ponto tem noção se está a fazer bem ou mal o seu trabalho?"), tendo os alfas de Chronbach sido de .87, .75 e .68, respetivamente. Estes valores são similares aos obtidos em estudos com a versão usada em amostras de trabalhadores portugueses (e.g., Afonso, 2011; Neiva, 2011). Por exemplo, no estudo de Neiva (2011), os valores de alfa de Chronbach obtidos foram de .80 ("Autonomia"), .78 ("Variedade de Competências") e .69 ("Feedback").

No âmbito da avaliação das características do trabalho pretendia-se ainda avaliar o ritmo de trabalho dos operadores de call-center. Uma vez que não foi encontrada uma escala validada para o contexto português que medisse este constructo, optou-se por criar uma escala para conhecer a perceção de cadência de trabalho que os operários de uma organização estão sujeitos no decorrer da sua função a partir de alguns dos itens presentes na 6. $\underline{\text { a }}$ edição do Inquérito Europeu Sobre as Condições de Trabalho (Eurofound, 2016). Especificamente, foram considerados seis itens que avaliam o ritmo de trabalho e dois itens que avaliam a duração e o intervalo médio entre chamadas. Assim e, relativamente ao ritmo de trabalho, os seis itens que compõem a medida são: i) "Permanecer sentado"; ii) "Movimentos repetitivos da mão ou do braço"; iii) "Trabalhar com computadores, portáteis, smatphones, etc."; iv) "Velocidade de trabalho elevada"; v) "Trabalhar em função de prazos rígidos" e vi) "O meu trabalho implica tarefas breves e repetitivas". Estes itens foram avaliados numa escala tipo Likert, de sete pontos, variando entre (1) "Nunca" e (7) "Todo o tempo". Dado que esta medida foi desenvolvida no âmbito do presente estudo, será alvo da análise das suas propriedades psicométricas, análise essa que será apresentada na secção dos Resultados. Às duas questões cujo objetivo era conhecer o tempo médio de duração de uma chamada e o tempo médio de descanso dos operadores entre chamadas, as respostas eram dadas com base numa escala de Likert de seis pontos, que variava entre (1) "Menos de 3 minutos" e (6) "Mais de 15 minutos".

Fatores de Stress Ocupacional. Para avaliar os fatores de stress foi utilizado o Questionário de Stress Ocupacional - Versão Geral (QSO-VG; Gomes, 2010), tendo este sido usado em várias amostras portuguesas de trabalhadores (e.g., Baganha et al., 2016; Carvalho \& Lobo, 2015). Este instrumento pretende avaliar as potenciais fontes indutoras de stress, sendo tais fontes avaliadas por 24 itens numa escala de Likert que varia entre (0) "Nenhum stress" e (4) "Elevado Stress". Estes itens estão distribuídos por sete subescalas: "Relação com os utentes", que descreve o stress relacionado com as pessoas a quem o profissional presta serviços (e.g., "Cumprir com aquilo que é esperado pelas pessoas a quem presto os meus serviços (ex: clientes, cidadãos, etc.)"; "Relação com as chefias", que refere o stress relacionado com a relação do profissional com os superiores hierárquicos (e.g., "A falta de apoio e ajuda por parte dos meus superiores"); "Relação com os colegas", que apresenta o stress relacionado com a relação do profissional com os colegas de trabalho (e.g., "Conflitos e problemas com colegas de trabalho"; "Excesso de trabalho", que avalia o stress do profissional relacionado com a carga de trabalho e com o número de horas de trabalho a realizar (e.g., "Trabalhar muitas horas seguidas"); "Carreira e remuneração", que descreve o stress do profissional relacionado com as perspetivas de desenvolvimento da carreira profissional e salário recebido (e.g., "A falta de possibilidades de desenvolvimento e promoção na carreira”); "Problemas familiares", que se refere ao stress do profissional relacionado com o relacionamento familiar e apoio por parte de pessoas significativas (e.g., "Falta de tempo para manter uma boa relação com as pessoas mais próximas (ex: cônjuge, filhos, amigos, etc.)"; e "Condições de trabalho", que apresenta o stress dos profissionais relacionado com os meios humanos e materiais disponíveis para a realização adequada das tarefas profissionais (e.g., "Falta de condições de trabalho").

Os valores de alfa de Cronbach obtidos por cada subescala foram: .77 (Relação com utentes), .79 (Relação com chefias), .87 (Excesso de trabalho), .86 (Relação com colegas), .81 (Carreira e remuneração), .92 (Problemas familiares) e .84 (Condições de trabalho).

Compromisso Organizacional. Na avaliação do compromisso organizacional foi usada a versão portuguesa (Nascimento et al., 2008) da Escala de Compromisso Organizacional de Meyer e Allen (1997). 
Esta versão inclui 19 itens, sendo solicitado ao participante para avaliar o seu grau de concordância com as afirmações que lhe são apresentadas, numa escala de Likert de sete pontos, variando entre (1) "Discordo totalmente" e (7) "Concordo totalmente". De referir que pontuações mais elevadas correspondem a maior compromisso com a organização. Estes itens estão organizados em três subescalas: "Compromisso afetivo", constituída por seis itens que descrevem o nível de compromisso dos operadores com a organização através de relações emocionais (e.g., "Não me sinto "emocionalmente ligado" a esta empresa"); "Compromisso calculativo", composta por sete itens, apresenta o nível de compromisso resultante das relações transacionais apoiadas num investimento pessoal que visa um determinado retorno (e.g., "Acredito que há muito poucas alternativas para poder pensar em sair desta empresa"; e "Compromisso normativo", formada por seis itens, representa o compromisso com a organização através de sentimentos de obrigação e de dever moral (e.g., "Sinto que não tenho qualquer dever moral em permanecer na empresa onde estou atualmente"). Os valores de alfa de Cronbach para a versão portuguesa foram de .91 (Compromisso afetivo), .84 (Compromisso normativo) e de .91 (Compromisso calculativo).

No presente estudo foi também analisada a fiabilidade das subescalas através dos alfas de Cronbach tendo sido obtidos valores de .82 (Compromisso afetivo), .65 (Compromisso Calculativo) e .84 (Compromisso normativo).

Espaço para sugestões e comentários. No final do protocolo de investigação existia ainda um espaço para os participantes deixarem algum comentário/sugestão ou referirem algum ponto que considerassem relevante em relação à experiência que tinham como operadores de call-center, caso assim o desejassem.

\section{Procedimento}

Depois de construído o protocolo de investigação em versão online e em versão papel, foi realizado um primeiro pré-teste. Nesta fase foram contactados alguns operadores de call-center, explicando o objetivo do estudo. Após aceitarem participar neste pré-teste, o protocolo online foi enviado por e-mail a três operadores de call-center (inbound e outbound) que responderam, por e-mail e por entrevista telefónica, com algumas observações e recomendações ao nível de estruturações frásicas dos enunciados. Introduzidas as alterações recomendadas, foi concretizado um segundo pré-teste, tendo nele participado seis operadores de call-center (inbound e outbound), os quais não sugeriram qualquer tipo de alteração.

De seguida, contactou-se um call-center de uma operadora de telecomunicações localizado no distrito do Porto, tendo sido explicitados os objetivos e pertinência do estudo, bem como os respetivos procedimentos, enviando, em anexo, o link da versão online apresentando a forma como os dados seriam recolhidos. Depois de aprovada a recolha de dados na organização, o link do protocolo foi enviado por $e$ mail a todos os operadores inbound e outbound. No corpo de e-mail foram descritas informações acerca do estudo assim como do preenchimento do questionário. Todavia, devido à fraca adesão a tal formato (64 respostas, 15 inbound e 49 outbound), optou-se por recorrer ao formato em papel.

Relativamente à recolha de dados em papel, esta implicou três deslocações à organização: uma para conhecer a estrutura física da organização e presenciar chamadas inbound e outbound, outra para a entrega dos protocolos de investigação e a última para a recolha dos mesmos. Foram entregues, na primeira visita, 300 protocolos, correspondendo ao número de operadores existentes no call-center, tendo sido devolvidos 228 (taxa de resposta de 76\%). Foram também entregues cartas dirigidas às chefias diretas, uma vez que foram elas que efetuaram a distribuição dos questionários pelos operadores. Nessas cartas encontravam-se descritas as informações acerca do estudo, do preenchimento do protocolo e das condições de participação. Aos participantes foi também entregue um consentimento informado onde estas informações também eram apresentadas. Por forma a garantir o anonimato e a confidencialidade total dos dados recolhidos, foram fornecidos a cada operador dois envelopes endereçados à equipa de investigação, um para devolução do questionário e outro para o consentimento.

Procedimentos de análise dos dados. Para a análise dos dados quantitativos recorreu-se ao programa estatístico de análise de dados Statistical Package for the Social Sciences (IBM® SPSS®, versão 23.0) e foram realizadas análises descritivas e análises inferenciais, nomeadamente teste de diferenças entre os grupos em análise. Tendo em conta o cumprimento ou não dos pressupostos para a utilização de testes paramétricos (Martins, 2011), recorreu-se ao teste $t$ de Student ou ao teste de Mann-Whitney (U) para a análise de diferenças entre os grupos inbound e outbound. No entanto, em termos de apresentação dos resultados derivados de tais análises, seguimos a estratégia recomendada por Fife-Schaw (2006) e citada por Martins (2011), ou seja, quando os resultados dos testes paramétricos e não-paramétricos chegam exatamente às mesmas conclusões (i.e., em termos de aceitação ou rejeição da hipótese nula), optamos pela apresentação dos resultados dos testes paramétricos. 
As respostas dadas no espaço destinado às sugestões e comentários foram sujeitas a análise de conteúdo (Amado, 2000). Uma vez que a medida relativa ao "ritmo de trabalho" foi elaborada no contexto do presente estudo, a análise dos resultados iniciou-se, no entanto, pela análise das características psicométricas de tal medida.

\section{RESULTADOS}

Análise fatorial exploratória da medida "ritmo de trabalho"

Como referido, a análise dos dados iniciou-se pela análise fatorial exploratória dos componentes principais com rotação varimax dos seis itens que avaliavam o "ritmo de trabalho". 0 valor de KMO (Kaiser-Meyer-Olkin measure of sampling adequacy) obtido foi de .70, estando acima do valor recomendado (.50) para aceitação da amostra (Field, 2013). Também o teste de esfericidade de Bartlett permitiu a continuidade da análise, pois o resultado obtido foi significativo, $\chi^{2}(15)=230.84, p<.001$.

Desta análise extraíram-se dois fatores que explicavam $56.34 \%$ da variância total. A Tabela 3 apresenta os resultados dessa análise fatorial, com os pesos de saturação obtidos por cada item em cada fator extraído, bem como os resultados da análise de fiabilidade (alfa de Cronbach) realizada para os dois fatores.

Tabela 3. Resultados da análise fatorial exploratória para os itens relativos à Organização e Ritmo de Trabalho e da análise de fiabilidade para os fatores extraídos

\begin{tabular}{lcc}
\hline \multirow{2}{*}{ Itens } & \multicolumn{2}{c}{ Fatores } \\
\cline { 2 - 3 } & $\mathbf{1}$ & $\mathbf{2}$ \\
\hline Permanecer sentado & .179 & $\mathbf{. 7 3 0}$ \\
Movimentos repetitivos da mão ou do braço & .465 & $\mathbf{. 4 6 8}$ \\
Trabalhar com computadores, portáteis, smartphones, etc. &. .077 &. $\mathbf{7 1 1}$ \\
Velocidade de trabalho elevada & $\mathbf{. 7 9 4}$ & .113 \\
Trabalhar em função de prazos rígidos & $\mathbf{. 8 4 0}$ & -.137 \\
O meu trabalho implica tarefas breves e repetitivas & $\mathbf{. 6 1 0}$ & .359 \\
\hline \% de variância explicada por fator & 32.7 & 23.63 \\
Alfa de Cronbach & .66 & .40 \\
\hline
\end{tabular}

Tendo em consideração que o item "Movimentos repetitivos da mão ou do braço" teve valores de saturação abaixo de .50, de acordo com o critério recomendado por Pestana e Gageiro (2008), este foi excluído. A exclusão deste item resultou num fator com apenas dois itens o que, de acordo com Costello e Osborne (2005), tornava tal fator fraco e instável uma vez que tem menos de três itens. Por outro lado, o valor do alfa de Cronbach obtido (.40) é também considerado inaceitável (Field, 2013). Assim, foi retido o primeiro fator constituído por três itens ao qual foi dado o nome de "Ritmo de trabalho". Na cotação desta escala, quanto maior o valor obtido maior a perceção do ritmo de trabalho.

\section{Análise descritiva}

As análises descritivas para cada variável em estudo são apresentadas na Tabela 4.

Relativamente às características do trabalho, os resultados apontam para uma baixa autonomia percecionada pelos trabalhadores assim como para um elevado ritmo de trabalho. A relação com a carreira e a remuneração foi reportada como a principal fonte de stress. Por sua vez, no que concerne ao compromisso organizacional, os operadores mostraram um nível médio de compromisso com a organização onde trabalham. 
Tabela 4. Medidas descritivas das variáveis em estudo

\begin{tabular}{lccc}
\hline Variáveis & $\begin{array}{c}\text { Média } \\
\text { (Desvio-padrão) }\end{array}$ & Min-Máx & Mediana \\
\hline Características do trabalho & $2.69(1.43)$ & $1.00-7.00$ & 2.33 \\
$\quad$ Autonomia & $3.62(1.15)$ & $1.00-7.00$ & 3.67 \\
$\quad$ Feedback & $3.57(1.40)$ & $1.00-7.00$ & 3.67 \\
$\quad$ Variedade de competências & $5.79(0.98)$ & $1.75-7.00$ & 6.00 \\
$\quad$ Ritmo de trabalho & & & 3.00 \\
Fontes de stress & $2.81(0.88)$ & $0.00-4.00$ & 2.75 \\
$\quad$ Carreira e Remuneração & $2.70(0.82)$ & $0.25-4.00$ & 2.33 \\
$\quad$ Relação com os utentes & $2.39(1.13)$ & $0.00-4.00$ & 1.00 \\
$\quad$ Problemas familiares & $1.21(1.01)$ & $0.00-4.00$ & 2.50 \\
$\quad$ Relação com colegas & $2.43(0.96)$ & $0.00-4.00$ & 1.33 \\
$\quad$ Excesso de trabalho & $1.52(0.98)$ & $0.00-4.00$ & 1.67 \\
$\quad$ Condições de trabalho & $1.77(1.04)$ & $0.00-4.00$ & 3.50 \\
$\quad$ Relação com as chefias & & & 4.00 \\
Compromisso organizacional & $3.52(0.90)$ & $1.33-6.00$ & 3.00 \\
$\quad$ Afetivo & $3.96(1.00)$ & $1.29-6.57$ & $1.00-7.00$ \\
$\quad$ Calculativo & $3.08(1.32)$ & & \\
Normativo & &
\end{tabular}

As medidas descritivas relativas à duração média de chamadas e ao intervalo médio existente entre chamadas são apresentadas na Tabela 5.

Tabela 5. Medidas descritivas relativas à duração e intervalo médio entre chamadas

\begin{tabular}{|c|c|c|c|c|c|c|}
\hline \multirow{2}{*}{ Variável } & \multicolumn{2}{|c|}{ Inbound } & \multicolumn{2}{|c|}{ Outbound } & \multicolumn{2}{|c|}{ Total } \\
\hline & $n$ & $\%$ & $n$ & $\%$ & $n$ & $\%$ \\
\hline \multicolumn{7}{|l|}{ Duração média de chamadas } \\
\hline inferior a 3 minutos & 8 & 4.2 & 9 & 11.0 & 17 & 6.2 \\
\hline entre 3 e 6 minutos & 67 & 35.1 & 46 & 56.1 & 113 & 41.2 \\
\hline entre 6 e 9 minutos & 62 & 32.5 & 14 & 17.1 & 76 & 27.7 \\
\hline entre 9 e 12 minutos & 30 & 15.7 & 10 & 12.2 & 40 & 14.6 \\
\hline entre 12 e 15 minutos & 18 & 9.4 & 1 & 1.2 & 19 & 6.9 \\
\hline superiores a 15 minutos & 6 & 3.1 & 2 & 2.4 & 8 & 2.9 \\
\hline \multicolumn{7}{|c|}{ Duração do intervalo médio entre chamadas } \\
\hline inferior a 3 minutos & 168 & 88.9 & 74 & 90.2 & 242 & 88.3 \\
\hline entre 3 e 6 minutos & 9 & 4.8 & 6 & 7.3 & 15 & 5.5 \\
\hline entre 6 e 9 minutos & 5 & 2.6 & 0 & 0 & 5 & 1.8 \\
\hline entre 9 e 12 minutos & 5 & 2.6 & 1 & 1.2 & 6 & 2.2 \\
\hline entre 12 e 15 minutos & 0 & - & 0 & - & 0 & - \\
\hline superiores a 15 minutos & 2 & 1.1 & 1 & 1.2 & 3 & 1.1 \\
\hline
\end{tabular}

Nota. Dada a presença de valores omissos em certas variáveis, o $\mathrm{n}$ obtido pode diferir do tamanho da amostra $(\mathrm{N}=274)$.

0 teste de Mann-Whitney revelou a existência de diferenças estatisticamente significativas entre o serviço inbound e outbound em relação à duração média de chamadas, $U=5501.00, p<.001$. Os participantes do serviço inbound (Ordem Média $=149.20$ ) relataram tempos médios de chamadas superiores aos participantes do serviço outbound (Ordem Média = 108.59).

Por outro lado, não foram observadas diferenças estatisticamente significativas entre os serviços inbound (Ordem Média $=136.66)$ e outbound (Ordem Média $=134.47)$ em relação ao intervalo entre chamadas, como revelaram os resultados do teste de Mann-Whitney, $U=7623.50, p=.69$. 


\section{Comparação entre serviços Inbound e Outbound}

Na Tabela 6 são apresentados os resultados resultantes da comparação das variáveis em estudo entre os serviços Inbound e Outbound.

Tabela 6. Resultados dos testes $t$-Student para a análise de diferenças entre os grupos Inbound e Outbound

\begin{tabular}{|c|c|c|c|c|}
\hline Variáveis & $\begin{array}{c}\text { Inbound } \\
\text { (n= 191) } \\
\text { Média (DP) }\end{array}$ & $\begin{array}{c}\text { Outbound } \\
\text { (n= 83) } \\
\text { Média (DP) }\end{array}$ & $d f$ & $t$ \\
\hline \multicolumn{5}{|l|}{ Características do trabalho } \\
\hline Autonomia & $2.73(1.40)$ & $2.61(1.52)$ & 269 & .59 \\
\hline Feedback & $3.60(1.14)$ & 3.67 (1.19) & 272 & -.51 \\
\hline Variedade de competências & $3.88(1.31)$ & $2.86(1.35)$ & 272 & $\mathbf{5 . 8 4}^{* * *}$ \\
\hline Ritmo de Trabalho & $5.82(0.96)$ & $5.73(1.03)$ & 270 & .76 \\
\hline \multicolumn{5}{|l|}{ Fontes de stress } \\
\hline Carreira e Remuneração & $2.83(0.92)$ & $2.78(0.82)$ & 268 & .41 \\
\hline Relação com os utentes & $2.72(0.82)$ & $2.70(0.83)$ & 269 & .43 \\
\hline Problemas familiares & $2.50(1.13)$ & $2.11(1.09)$ & 270 & $2.60^{* *}$ \\
\hline Relação com colegas & $1.14(1.04)$ & $1.35(0.93)$ & 272 & -1.55 \\
\hline Excesso de trabalho & $2.48(0.97)$ & $2.32(0.93)$ & 272 & 1.27 \\
\hline Condições de trabalho & $1.49(0.99)$ & $1.58(0.96)$ & 272 & -.67 \\
\hline Relação com as chefias & $1.83(1.07)$ & $1.60(0.99)$ & 272 & 1.69 \\
\hline \multicolumn{5}{|l|}{ Compromisso organizacional } \\
\hline Afetivo & 3.45 (1.35) & $3.55(1.18)$ & 272 & -.58 \\
\hline Calculativo & $3.93(1.03)$ & $4.05(0.92)$ & 272 & -.94 \\
\hline Normativo & 3.00 (1.37) & $3.26(1.20)$ & 272 & -1.54 \\
\hline
\end{tabular}

${ }^{*} p<.05 ;{ }^{* *} p<.01 ;{ }^{* * *} p<.001$

Em relação às características do trabalho, foram encontradas diferenças estatisticamente significativas entre os dois serviços relativamente à perceção de "Variedade de competências" existente no trabalho, sendo que os operadores do serviço inbound reportaram haver uma maior variedade de competências do que os participantes do serviço outbound.

Também relativamente às fontes de stress, apenas foram encontradas diferenças estatisticamente significativas entre os serviços de inbound e outbound ao nível dos "Problemas Familiares", sendo que, também neste caso, foram os operadores alocados ao serviço inbound que relataram mais stress resultante dos problemas familiares em comparação com os operadores do serviço outbound.

\section{Análise das sugestões e comentários}

Dos 274 operadores de call-center que constituíam a amostra, 33 (12\%) fizeram algum tipo de comentário na última seção do protocolo de investigação. Uma resposta foi excluída dado que não estava relacionada com o tema analisado. Assim, foram analisadas 32 respostas $(11.7 \%)$, sendo que a maioria pertencia ao serviço inbound ( $n=25,78.1 \%)$ e apenas sete $(21.9 \%)$ ao serviço outbound.

Uma grande parte dos operadores $(n=13,40.6 \%$; quatro outbound e nove inbound $)$ fez comentários relacionados com o baixo tempo de intervalo entre as chamadas e o desgaste psicológico inerente a este tipo de trabalho (e.g., "Só temos dez segundos entre chamadas. Evitem este trabalho ao máximo, escravatura do século XXI"). Também 12 participantes (37.5\%; dois outbound e dez inbound) fizeram referência a aspetos relacionados com a falta de progressão na carreira e a baixa remuneração (e.g., "Sendo operador de call-center há três anos o que mais me revolta é não existir a possibilidade de evoluir neste momento, e não é um trabalho de todo compensatório ao nível pessoal e monetário (...)"). Dois operadores (6.3\%; um outbound e um inbound) apontaram ainda como problema o terem poucas férias e descanso (e.g., "Gestão de férias e pausas incomportável"). Um participante (3.1\%), do grupo inbound, fez ainda referência a problemas relacionados com a relação com as chefias ("(...) uma vida de trabalho em call-center não compensa a não ser que sejamos namorados e "companheiros" e amiguinhos dos chefes para subir de posto") e outro participante (3.1\%), também do grupo inbound, apontou aspetos relacionados com as consequências do ritmo de trabalho ao nível de problemas familiares ("Trabalhar num call-center 
é um trabalho ao nível psicológico muito desgastante e stressante levando por vezes a não ter paciência para quando chegamos a casa aturar os nossos amigos, namorada e família"). De referir ainda que três participantes, do grupo inbound, indicaram aspetos positivos deste tipo de trabalho (e.g., "Para mim este trabalho não é mau, como muita gente fala eu pelo menos gosto daquilo que faço" e "gosto de "resolver" os problemas dos clientes").

\section{DISCUSSÃO}

O presente estudo teve como principais objetivos: realizar uma análise descritiva das características do trabalho, dos fatores de stress ocupacional e do nível de compromisso com a organização por parte dos operadores de um call-center português e, também, comparar os serviços inbound e outbound relativamente a estas variáveis para apurar se existiam diferenças significativas.

Relativamente ao primeiro objetivo, em termos descritivos, verificou-se que os operadores de callcenter revelavam ter pouca autonomia na realização de tarefas, recebiam algum feedback do seu trabalho e, ainda, tinham um elevado ritmo de trabalho. Estes dados são consistentes com estudos anteriores (e.g., Santos \& Marques, 2006; Venco, 2006), que mostraram que os operadores de call-center não têm autonomia para planear e organizar o trabalho de forma independente, estando sujeitos a algum feedback, e com a perceção de um ritmo de trabalho excessivo (Rose \& Wright, 2005).

Em relação às fontes de stress, os operadores de call-center apresentaram como principal fonte de stress a carreira e a remuneração, o que foi apoiado pela análise de conteúdo das sugestões/comentários de alguns operadores (e.g., “...não existir possibilidade de evoluir neste momento, e não é um trabalho de todo compensatório ao nível pessoal e monetário"). Este resultado não vai de encontro ao estudo de Lin et al. (2010) que apontava a "relação com os clientes" como a principal fonte de stress. Esta diferença pode estar relacionada com o contexto específico da organização estudada, tendo em conta que Lin et al. (2010) analisaram o contexto de trabalho em call-center numa instituição bancária.

Ainda, em termos descritivos, os resultados mostraram que os trabalhadores apresentavam um compromisso organizacional médio nas três dimensões. Estes dados são consistentes com estudos anteriores (e.g., Maia, 2011; Paiva et al., 2015), sendo que este compromisso organizacional pode não ser tão elevado devido ao descontentamento dos trabalhadores com a forma como o trabalho está organizado ou com o baixo capital psicológico, como sugeriram outros autores (e.g., Jaaron \& Backhouse, 2011; Simons \& Buitendach, 2013). Esta ideia foi também apoiada pelos comentários feitos pelos participantes no último tópico do protocolo, “(...) e não é um trabalho de todo compensatório ao nível pessoal e monetário").

Quanto ao segundo objetivo, no que diz respeito às características do trabalho, apenas foram encontradas diferenças entre os serviços ao nível da variedade de competências, com os participantes do serviço inbound a reportarem uma maior variedade de competências no seu trabalho do que os participantes do serviço outbound. Estas diferenças podem ser justificadas pelo facto dos trabalhadores do serviço inbound receberem chamadas e terem de lidar com um variado leque de situações, adaptandose ao discurso/problema do cliente, tendo assim que usar uma maior variedade de competências para lidar com um telefonema (Chuchual et al., 2010). Tal facto foi, ainda, confirmado pela análise qualitativa, sendo que um participante do grupo inbound apontou este como um aspeto positivo ("...gosto de "resolver" os problemas dos clientes"). Em relação ao ritmo de trabalho e ao tempo médio entre chamadas, não foram encontradas diferenças estatisticamente significativas entre os tipos de serviços, o que pode dever-se ao facto dos serviços de call-center considerarem mais importante a quantidade de chamadas efetuadas/atendidas do que propriamente a qualidade do serviço (Frenkel et al., 1998). Na possibilidade de ambos os serviços se regerem por este princípio, compreende-se a ausência de diferenças ao nível do ritmo de trabalho. No entanto, verificou-se que os participantes do serviço inbound relataram tempos médios de chamadas superiores aos do serviço outbound. Este resultado é congruente com a característica do tipo de serviço, uma vez que os operadores inbound precisam de mais tempo para resolver os problemas dos clientes, enquanto os operadores outbound têm como objetivo captar a atenção do cliente e vender um produto o mais rapidamente possível. Em relação às fontes de stress, verificou-se que os participantes do serviço inbound declararam ter mais stress ao nível dos problemas familiares do que os participantes do serviço outbound. Tendo em conta que os operadores do serviço inbound têm como função receber chamadas de clientes no sentido de resolver problemas, podem ficar mais suscetíveis aos problemas apresentados fora do contexto de trabalho, gerando problemas em termos de contexto familiar e social. Este resultado é consistente com o indicado por Roque (2008), que apresentou como principal problema a desmotivação dos operadores para a vida social e familiar causada pelos elevados níveis de exigência e de stress permanente e com o de Khalid et al. (2013), que apontou que a exposição à hostilidade por parte dos clientes como uma das principais fontes de stress. Esta ideia 
também foi defendida por um participante do serviço inbound “(...) levando por vezes a não ter paciência para quando chegamos a casa aturar os nossos amigos, namorada e família".

Ainda no contexto da discussão sobre as fontes de stress ocupacional, de referir também que além da "relação dos clientes" e dos "problemas familiares", tanto para a amostra global como em função do tipo de serviço (inbound ou outbound), as fontes que exibiriam valores médios acima do ponto intermédio da escala foram "carreira e remuneração" e "excesso de trabalho" o que, a par da baixa perceção de autonomia e elevado ritmo de trabalho, aponta, globalmente, para condições laborais que poderíamos classificar como exigentes do ponto de vista do bem estar e saúde ocupacional. Neste âmbito, importa ainda referir que tem sido observado o impacto de tais condições na saúde dos operadores de call center (e.g., Lin et al., 2010; Oh et al., 2017). Por exemplo, no estudo de Oh et al (2007), a variável identificada como mais crítica nesse impacto em termos de saúde mental foi a "dissonância emocional" exigida pelo trabalho (i.e., discrepância entre as emoções sentidas e as exibidas), levando os autores a recomendarem a necessidade de uma monitorização frequente desta dimensão no contexto dos call-center de modo a minimizar tal impacto.

Relativamente ao compromisso organizacional, não foram encontradas diferenças estatisticamente significativas entre os grupos, o que contraria os resultados obtidos por Echchakoui (2016) onde operadores inbound apresentaram uma menor identificação com a organização quando comparados com os operadores outbound.

$\mathrm{Na}$ interpretação destes resultados, devem ter-se em consideração algumas limitações. Em primeiro lugar, a possibilidade de enviesamento na recolha dos dados, pois a entrega e a recolha dos questionários foram efetuadas pelos responsáveis das equipas da organização contactada, o que não permitiu controlar a forma de distribuição dos questionários junto dos operadores. Também, os protocolos não foram aplicados todos da mesma forma, alguns foram aplicados em formato online, enquanto outros (a maioria neste estudo) foram aplicados em formato papel. Deve-se também ter em conta a relativa assimetria no número de participantes de cada serviço, sendo que a maioria se encontrava no serviço inbound. Ao nível do ritmo de trabalho, teria sido interessante medir o número de chamadas atendidas por cada participante do grupo inbound e outbound. Por fim, a amostra deste estudo foi bastante homogénea na medida que se restringe a um contexto muito específico dentro do trabalho em call-center. No futuro, seria importante replicar este estudo com amostras mais heterogéneas incluindo grupos distintos dentro do contexto de trabalho em call-center, permitindo uma maior generalização dos resultados.

Apesar das limitações referidas, e tendo em conta que poucos estudos têm comparado o serviço inbound e outbound ao nível do impacto deste tipo de serviços nos operadores, este foi, tanto quanto é do nosso conhecimento, o primeiro estudo em contexto português a fazer esta comparação incluindo várias dimensões relacionadas com o contexto de trabalho dos operadores de call-center. Assim, este estudo vem enriquecer a compreensão deste tipo de trabalho e das suas repercussões nos operadores dos serviços inbound e outbound em contexto português. Dadas as perspetivas de crescimento deste modo de organização do trabalho no contexto nacional e internacional (e.g. Altitude, 2015, 2016; Costa \& Costa, 2018; Roque, 2008), reforça ainda mais a relevância do aprofundamento da investigação nesta área.

\section{REFERÊNCIAS}

Afonso, M. M. (2011). O modelo das características do trabalho o compromisso organizacional à luz da troca social (Dissertação de Mestrado não publicada). Escola de Psicologia, Universidade do Minho, Braga.

Alcover, C. M., Íñigo, D. M., \& Chambel, M. J. (2012). Perceptions of employment relations and permanence in the organization: Mediating effects of affective commitment in relations of psychological contract and intention to quit. Psychological Reports, 110(3), 839-853. https://doi.org/10.2466/01.07.21.PR0.110.3.839-853.

Altitude (2015). European Contact center Benchmark Platform Whitebook 2014. http://www.aprocs.pt/activeapp/wpcontent/uploads/2015/11/Altitude_European_CC_Benchmark_ExecSummary.pdf.

Altitude (2016). International customer contact benchmark 5th edition. https://www.altitude.com/downloads/international-customer-contact-benchmark-2016infographic.pdf.

Amado, J. (2000). A técnica de análise de conteúdo. Revista Referência, 5, 53-63.

Baganha, C., Gomes, A. R., \& Esteves, A. (2016). Stresse ocupacional, avaliação cognitiva, burnout e comprometimento laboral na aviação civil. Psicologia, Saúde \& Doenças, 17(2), 265-281. https://doi.org/10.15309/16psd170212 
Boonzaier, B., Ficker, B., \& Rust, B. (2001). A review of research on the job characteristics model and the attendant job diagnostic survey. South African Journal of Business Management, 32, 1-24.

Carvalho, J. P. M. T., \& Lobo, F. (2015). Experiências de recuperação e o stress ocupacional em psicólogos(as), no exercício da sua função. Revista de Estudios e Investigación en Psicología y Educación, Extr., 14, 077-085. https://doi.org/10.17979/reipe.2015.0.14.941.

Chuchual, P., Chongpravatisakul, N., Kusolmanomai, T., \& Komolavanij, S. (2010, junho). Inbound and outbound calls assignment for an efficient call center. Artigo apresentado na $7^{\text {th }}$ International Conference on Service Systems and Service Management, Tóquio. https://doi.org/10.1109/ICSSSM.2010.5530196.

Costa, H. A, \& Costa, E. S. (2018). Trabalho em call centers em Portugal e no Brasil a precarização vista pelos operadores. Tempo Social,10(1), 105-127. https://doi.org/10.11606/01032070.ts.2018.123181.

Costello, A. B., \& Osborne, J. (2005). Best practices in exploratory factor analysis: Four recommendations for getting the most from your analysis. Practical Assessment Research \& Evaluation, 10(73), 1-9. https://doi.org/10.1.1.110.9154.

Dhanpat, N., Modau, F. D., Lugisani, P., Mabojane, R., \& Phiri, M. (2018). Exploring employee retention and intention to leave within a call centre. SA Journal of Human Resource Management, 16(1), 1-13. https://doi.org/10.4102/sajhrm.v16i0.905

Echchakoui, S. (2016). Addressing differences between inbound and outbound agents for effective call center management. Global Business and Organizational Excellence, 36(1), 71-86. https://doi.org/10.1002/joe.21757.

Eurofound (2016). Inquérito Europeu Sobre as Condições de Trabalho. http://www.eurofound.europa.eu/sites/default/files/page/field_ef_documents/pt_questionnaire. pdf.

Field, A. P. (2013). Discovering statistics using IBM SPSS Statistics: And sex and drugs and rock ' $n$ ' roll ( $4^{\text {th }}$ ed.). Sage.

Frenkel, S., Tam, M., Korczynski, M., \& Shire, K. (1998). Beyond bureaucracy? Work organization in call centres. The International Journal of Human Resource Management, 9(6), 957-979. https://doi.org/10.1080/095851998340694.

Gomes, A. R. (2010). Questionário de Stresse Ocupacional - Versão Geral (QSO-VG) (Relatório técnico não publicado). Universidade do Minho, Escola de Psicologia, Braga.

Hackman, J. R., \& Oldham, G. R. (1976). Motivation through the design of work: Test of a theory. Organizational Behavior and Human Performance, 16, 250-279. https://doi.org/10.1016/00305073(76)90016-7.

Harvey, R., Billings, R., \& Nilan, K. (1985). Confirmatory factor analysis of the Job Diagnostic Survey: Good news and bad news. Journal of Applied Psychology, 70(3), 461-468. https://doi.org/10.1037/00219010.70.3.461

Holman, D. (2002). Employee wellbeing in call centres. Human Resource Management Journal, 12(4), 3550. https://doi.org/10.1111/j.1748-8583.2002.tb00076.x.

Holman, D. (2003). Call centres. In D. J. Holman, T. D. Wall, C. W. Clegg, P. Sparrow, \& A. Howard (Eds.), The new workplace: A guide to the human impact of modern working practices (pp. 115-134). Wiley.

Holman, D., Chissick, C., \& Totterdell, P. (2002). The effects of performance monitoring on emotional labor and well-being in call centers. Motivation and Emotion, 26(1), 57-81. https://doi.org/10.1023/A:1015194108376.

Idaszak, J. R., \& Drasgow, F. (1987). A Revision of the Job Diagnostic Survey: Elimination of a measurement artifact. Journal of Applied Psychology, 72(1), 69-74.

Jaaron, A. A. M., \& Backhouse, C. J. (2011). Systems thinking for call centre service design: Affective commitment implications in manufacturing enterprises. The Service Industries Journal, 31(4), 613628. https://doi.org/10.1080/02642069.2010.504301.

Khalid, A., Sarfaraz, A., Ahmed, S., \& Malik, F. (2013). Prevalence of stress among call center employees. Pakistan Journal of Social and Clinical Psychology, 11(2), 58-62.

Legros, B., Jouini, O., \& Koole, G. (2018). Blended call center with idling times during the call service. IISE Transactions, 50(4), 279-297. https://doi.org/10.1080/24725854.2017.1387318

Lin, Y.-H., Chen, C.-Y., Hong, W.-H., \& Lin, Y.-H. (2010). Perceived job stress and health complaints at a bank call center: Comparison between inbound and outbound services. Industrial Health, 48(3), 349-356. https://doi.org/10.2486/indhealth.48.349.

Lorente, L., \& Yeves, J. (2016). Modelos explicativos do stress laboral. In M. J. Chambel (Coord.), Psicologia da saúde ocupacional (pp. 71-94). Pactor. 
Maia, V. H. M. (2011). Comprometimento organizacional nos operadores de call center: Um estudo de caso (Dissertação de Mestrado não publicada). Instituto Superior de Economia e Gestão, Universidade de Lisboa, Lisboa.

Martins, C. (2011). Manual de Análise de dados quantitativos com recurso ao IBM SPSS: Saber decidir, fazer, interpretar e redigir. Psiquilíbrios Edições.

Meyer, J. P., \& Allen, N. J. (1991). A three-component conceptualization of organizational commitment. Human Resources Management Review, 1(1), 61-89. https://doi.org/10.1016/10534822(91)90011-Z.

Meyer, J. P., \& Allen, N. J. (1997). Commitment in the workplace: Theory, research, and application. Sage Publications, Inc.

Moreira, V., Alves, P. M., \& Botelho, M. C. (2015). Desumanização do trabalho e turnover num call center de uma instituição bancária. International Journal on Working Conditions, 10, 1-22. http://ricot.com.pt/PT/jornal.php?subop=0\&search=undefined\&language=PT.

Mustosmäki, A., Anttila, T., \& Oinas, T. (2013). Engaged or not? A comparative study on factors inducing work engagement in call center and service sector work. Nordic Journal of Working Life Studies, 3(1), 49-67. https://doi.org/10.19154/njwls.v3i1.2520

Nascimento, J. L., Lopes, A., \& Salgueiro, M. F. (2008). Estudo sobre a validação do "Modelo de Comportamento Organizacional" de Meyer e Allen para o contexto português. Comportamento Organizacional e Gestão, 14(1), 155-133). http://www.scielo.mec.pt/scielo.php?script=sci_serial\&pid=0872-9662\&lng=pt.

Neiva, F. 0. (2011). Efeitos da rotação dos postos de trabalho nos trabalhadores e na organização (Dissertação de Mestrado não publicada). Escola de Engenharia, Universidade do Minho, Guimarães.

Oh, H., Park, H., \& Boo, S. (2017). Mental health status and its predictors among call center employees: A cross-sectional study. Nursing \& Health Sciences, 19(2), 228-236. https://doi.org/10.1111/nhs.12334

Paiva, K. C. M., Dutra, M. R., \& Luz, T. R. (2015). Comprometimento organizacional de trabalhadores de call center. Revista de Administração, 50(3), 310-324. https://doi.org/10.5700/rausp1202.

Pestana, M. H., \& Gajeiro, J. N. (2008). Análise de dados para ciências sociais - A complementaridade do SPSS (5a Ed.). Edições Silabo, Lda.

Rod, M., \& Ashill, N. J. (2013). The impact of call centre stressors on inbound and outbound call-centre agent burnout. Managing Service Quality: An International Journal, 23(3), 245-264. https://doi.org/10.1108/09604521311312255.

Roque, I. (2008, junho). Trabalho e precariedade no sector das telecomunicações: Uma experiência local num quadro transnacional. Artigo apresentado no VI Congresso Português de Sociologia, Lisboa. http://www.aps.pt/vicongresso/.

Roque, I. (2013). A precariedade dos estudantes académicos no universo dos call centres. International Journal on Working Conditions, 6, 18-36.

Rose, E., \& Wright, G. (2005). Satisfaction and dimensions of control among call centre customer service representatives. Journal of Human Resource Management, 16(1), 136-160. https://doi.org/10.1080/0958519042000295000.

Sá, A. C. A. G. P., \& Sá, P. H. F. L. M. (2014). As características da função e seu impacto nos níveis de satisfação do funcionário de contato: Um estudo em uma grande empresa de telecomunicações. Revista Brasileira de Gestão de Negócios, 16(53), 658-676. https://doi.org/10.7819/rbgn.v16i52.1553.

Santos, M. J., \& Marques, A. P. (2006). 0 caso dos call centres: Organização do trabalho e atitudes face ao trabalho e emprego. Sociologia, Problemas e Práticas, 52, 67-86.

Scolari, C., Costa, S. G., \& Mazzilli, C. (2009). Prazer e sofrimento entre os trabalhadores de call-center. Psicologia USP, 20(4), 555-576. https://doi.org/10.1590/S0103-65642009000400005.

Silva, I., \& Keating, J. (2011). Escala de diagnóstico das características do trabalho (Relatório não publicado). Escola de Psicologia, Universidade do Minho, Braga.

Simons, J., \& Buitendach (2013). Psychological capital, work engagement and organisational commitment amongst call centre employees in South Africa. SA Journal of Industrial Psychology/SA Tydskrif vir Bedryfsielkunde, 39(2),1-12. https://doi.org/10.4102/sajip.v39i2.1071

Taylor, F. W. (1911/1998). The principles of a cientific management. Harper \& Bros.

Venco, S. (2006). Centrais de atendimento: A fábrica do século XIX nos serviços do século XXI. Revista Brasileira de Saúde Ocupacional, 31(114), 7-18. https://doi.org/10.1590/S030376572006000200002 
Vilela, L. V. D. O., \& Assunção, A. Á. (2004). Os mecanismos de controle da atividade no setor de teleatendimento e as queixas de cansaço e esgotamento dos trabalhadores. Cadernos de Saúde Pública, 20(4), 1069-1078. https://doi.org/10.1590/S0102-311X2004000400022

Wu, C. C., \& Liu, N. T. (2014). Perceived organizational support, organizational commitment and serviceoriented organizational citizenship behaviors. International Journal of Business and Information, 9(1), 61-88.

Zapf, D., Isic, A., Bechtoldt, M., \& Blau, P. (2003). What is typical for call centre jobs? Job characteristics, and service interactions in different call centres. European Journal of Work and Organizational Psychology, 12(4), 311-340. https://doi.org/10.1080/13594320344000183

Ziliotto, D. M., \& Oliveira, B. O. D. (2014). A organização do trabalho em call centers: Implicações na saúde mental dos operadores. Revista Psicologia Organizações e Trabalho, 14(2), 169-179.

Historial do artigo

Recebido $\quad 03 / 2019$

Aceite $\quad 07 / 2020$

Publicado $\quad 08 / 2020$ 